\title{
Impacto de Stents Farmacológicos em Pacientes com Doença Arterial Coronária Estável Submetidos a Intervenção Coronária Percutânea na Prática Diária do Mundo Real
}

\author{
Sergio Tavares ${ }^{1}$, Amanda G. M. R. Sousa', Ricardo A. Costa ${ }^{1}$, Adriana Moreira', J. Ribamar Costa Jr. ${ }^{1}$, \\ Galo Maldonado', Manoel Cano', Edson Romano', Enilton T. do Egito', Fausto Feres ${ }^{1}$, Ricardo Pavanello', \\ Daniel Magnoni', Cesar Jardim', Alexandre Abizaid'1', J. Eduardo Sousa'
}

\section{RESUMO}

Introdução: Estudos recentes incluindo pacientes portadores de doença coronária estável sugerem ausência de benefício clínico da estratégia inicial de intervenção coronária percutânea com stents não-farmacológicos associada a terapia medicamentosa otimizada vs. terapia medicamentosa otimizada isolada. No entanto, o tratamento ideal para pacientes com doença coronária estável no mundo real permanece controverso. Reportamos o impacto dos stents farmacológicos (SF) em pacientes portadores de doença coronária estável tratados na prática clínica diária. Método: Entre maio de 2002 e novembro de 2009, 1.814 pacientes portadores de doença coronária estável (angina estável ou isquemia silenciosa) foram prospectivamente incluídos no Registro DESIRE, e o seguimento clínico de até 8 anos (média de 3,9 \pm 2 anos) foi realizado em $98 \%$ desses pacientes. Resultados: A média das idades era de $64,2 \pm 10,8$ anos, $28 \%$ tinham diabetes melito, $21 \%$ tinham infarto do miocárdio prévio e $51,7 \%$, revascularização prévia. A artéria descendente anterior foi o vaso-alvo mais tratado $(42 \%)$, e, considerando-se o total de lesões $(n=2.701), 66,3 \%$ delas eram de elevada complexidade (tipos B2/C). Ao final do procedimento, o sucesso angiográfico foi de $99 \%$. No seguimento clínico tardio, as taxas cumulativas de eventos adversos foram: morte cardíaca, 3,4\%; infarto do miocárdio, 5,5\%; revascularização da lesão-alvo (RLA), 4,8\%; e trombose de stent, $0,8 \%$. Conclusão: A utilização de SF na doença coronária estável esteve associada a excelente evolução clínica tardia, incluindo taxa cumulativa de RLA $<5 \%$, e segurança sustentada. Comparados a dados históricos, tais achados demonstram grande benefício dos SF nessa população, sugerindo que a intervenção coronária percutânea

\section{ABSTRACT}

Impact of Drug Eluting Stents in Patients
with Stable Coronary Artery Disease Undergoing
Percutaneous Coronary Intervention in
Real World Clinical Practice

Background: Recent studies in patients with stable coronary artery disease suggest there is no clinical benefit of the initial strategy of percutaneous coronary intervention with bare metal stents associated to optimal medical therapy (OMT) vs OMT alone. However, the best therapeutic option for patients with stable coronary artery disease in the real world remains controversial. We report the impact of drug eluting stents (DES) in patients with stable coronary artery disease treated in the daily clinical practice. Method: From May 2002 to November 2009, 1,814 patients with stable coronary artery disease (stable angina or silent ischemia) were prospectively included in the DESIRE Registry and 98\% of the patients were followed up to 8 years (mean $3.9 \pm$ 2 years). Results: Mean age was $64.2 \pm 10.8$ years, $28 \%$ had diabetes mellitus, $21 \%$ had a prior myocardial infarction and $51.7 \%$ had a prior revascularization. Left anterior descending artery was treated in $42 \%$ of the patients. When all of the lesions ( $n=2,701$ ) were taken into consideration, $66.3 \%$ were classified as complex B2/C lesions. Angiographic success was $99 \%$. The cumulative rate of adverse events in the late clinical follow-up was: cardiac death, $3.4 \%$; myocardial infarction, 5.5\%; target-lesion revascularization (TLR), 4.8\%; and stent thrombosis, $0.8 \%$. Conclusion: The use of DES in stable coronary artery disease was associated to excellent late clinical follow-up including a cumulative TLR rate $<5 \%$ and stent thrombosis $<1 \%$. When compared to historical data, such findings show a great benefit of DES in this

\footnotetext{
1 Hospital do Coração - Associação do Sanatório Sírio - São Paulo, SP, Brasil.

Correspondência: Ricardo A. Costa. Rua Abílio Soares, 250 - 12ำ andar

- Paraíso - São Paulo, SP, Brasil - CEP 04005-000

E-mail: ricardoacosta@cardiol.br

Recebido em: 27/9/2010 • Aceito em: 20/11/2010
} 
Tavares S, et al. Impacto de Stents Farmacológicos em Pacientes com Doença Arterial Coronária Estável Submetidos a Intervenção Coronária Percutânea na Prática Diária do Mundo Real. Rev Bras Cardiol Invasiva. 2010;18(4):392-9.

com SF pode ser uma alternativa inicial segura e eficaz no tratamento de pacientes com doença coronária estável.

DESCRITORES: Doença das coronárias. Stents farmacológicos. Angioplastia transluminal percutânea coronária.

E studos recentes incluindo pacientes portadores de doença coronária estável sugerem ausência de benefício clínico da estratégia inicial de intervenção coronária percutânea com stents não-farmacológicos associada a terapia medicamentosa otimizada vs. terapia medicamentosa otimizada isolada. ${ }^{1}$ Embora esse achado venha causando impacto significativo na prática clínica, ${ }^{2}$ o tratamento ideal de pacientes com doença coronária estável no mundo real permanece controverso.

Considerando os stents farmacológicos, vários ensaios randomizados prévios demonstraram superioridade dos stents farmacológicos em relação aos stents nãofarmacológicos no tratamento de lesões coronárias em inúmeros cenários clínicos, em decorrência principalmente de reduções significativas de formação de hiperplasia neointimal, reestenose, e necessidade de revascularização da lesão-alvo. ${ }^{3-5}$ Digno de nota é o fato de que estudos com seguimento a longo prazo têm demonstrado efetividade clínica sustentada dos stents farmacológicos, sem comprometimento da segurança tardia, comparativamente aos stents não-farmacológicos. ${ }^{5,6}$ Mesmo assim, o impacto dos stents farmacológicos em pacientes com doença coronária estável no mundo real permanece desconhecido.

Logo, o objetivo de nossa análise foi avaliar o seguimento clínico tardio em pacientes portadores de doença coronária estável tratados com estratégia inicial de intervenção coronária percutânea com implante de stents farmacológicos na prática clínica do mundo real incluídos no registro Drug-Eluting Stent In the REal World (Registro DESIRE).

\section{MÉTODO}

\section{Protocolo e população estudada}

Os detalhes do protocolo e os resultados clínicos iniciais do Registro DESIRE já foram reportados previamente. ${ }^{7}$ De forma resumida, o Registro DESIRE é um estudo clínico prospectivo, não-randomizado, de braço único, com inclusão consecutiva de pacientes (inclusão em andamento), realizado em uma única instituição (Hospital do Coração da Associação do Sanatório Sírio - São Paulo, SP, Brasil). O estudo tem como objetivo investigar a evolução clínica tardia de pacientes tratados com stent farmacológico. A partir de maio de 2002, a intervenção coronária percutânea com stent farmacológico tem sido utilizada como estratégia preferencial nos pacientes encaminhados para tratamento percutâneo de rotina ou urgência na referida institui- population, suggesting that percutaneous coronary intervention with DES is a safe and effective initial strategy in patients with stable coronary artery disease.

KEY-WORDS: Coronary disease. Drug-eluting stents. Angioplasty, transluminal, percutaneous coronary.

ção. Foram incluídos pacientes com pelo menos uma lesão com estenose acima de $50 \%$ e anatomia favorável para intervenção coronária percutânea. Pelo protocolo, não existem limitações no que se refere ao número de lesões-alvo e/ou vasos-alvo que podem ser tratados ou ao número de stents farmacológicos implantados. O estudo está em consonância com a Declaração de Helsinque no que se refere à investigação em humanos, e foi aprovado pelo Comitê de Ética da referida instituição.

A doença coronária estável foi definida como apresentação clínica de angina estável (classificada pela Canadian Cardiovascular Society $-(\mathrm{CCS})^{8}$ ou isquemia silenciosa. Entre maio de 2002 e novembro de 2009, 3.534 pacientes foram prospectivamente incluídos no Registro DESIRE; desse total, 1.814 pacientes eram portadores de doença coronária estável e tinham, no mínimo, um ano de seguimento clínico (pacientes elegíveis), os quais compuseram a população de nossa subanálise (Figura 1).

\section{Procedimento}

De maneira geral, as intervenções coronárias percutâneas foram realizadas de acordo com as diretrizes atuais, ${ }^{9}$ e a estratégia final do procedimento foi deixada a critério do operador. Os seguintes stents farmacológicos de primeira e segunda gerações foram utilizados: Cypher ${ }^{\circledR}$, liberador de sirolimus (Cordis Corporation, Warren, Estados Unidos); $\operatorname{Taxus}^{\circledR}$, liberador de paclitaxel (Boston Scientific, Natick, Estados Unidos); Endeavor ${ }^{\circledR}$ e Resolute ${ }^{\circledR}$, liberadores de zotarolimus (Medtronic, Santa Rosa, Estados Unidos); Xience $\mathrm{V}^{\circledR}$ e Promus ${ }^{\circledR}$, liberadores de everolimus (Abbott Vascular, Santa Clara, Estados Unidos, e Boston Scientific, Natick, Estados Unidos, respectivamente), e BioMatrix ${ }^{\circledR}$, liberador de biolimus A9 (Biosensors International, Cingapura). Quando o resultado angiográfico imediato após o implante do stent demonstrava expansão subótima, era realizada pós-dilatação com cateter-balão de extensão mais curta que o stent, o qual era posicionado dentro das bordas do stent e insuflado a altas pressões (> 14 atm). Também eram permitidos implante de múltiplos stents e/ou tratamento de múltiplas lesões (por meio de procedimento estagiado ou não). O protocolo antitrombótico consistia na administração de dois agentes antiplaquetários: ácido acetilsalicílico e clopidogrel. O pré-tratamento (ácido acetilsalicílico 500 mg e clopidogrel 300-600 mg) foi realizado 24 horas antes da intervenção. Após a intervenção, a terapia com ácido acetilsalicílico era mantida 
Tavares S, et al. Impacto de Stents Farmacológicos em Pacientes com Doença Arterial Coronária Estável Submetidos a Intervenção Coronária Percutânea na Prática Diária do Mundo Real. Rev Bras Cardiol Invasiva. 2010;18(4):392-9.

Pacientes com $>18$ anos, com indicação de ICP de rotina ou urgência, com pelo menos 1 lesão coronária com obstrução $>50 \%$ favorável para implante de stent ( $\mathrm{SF}$ como estratégia inicial)

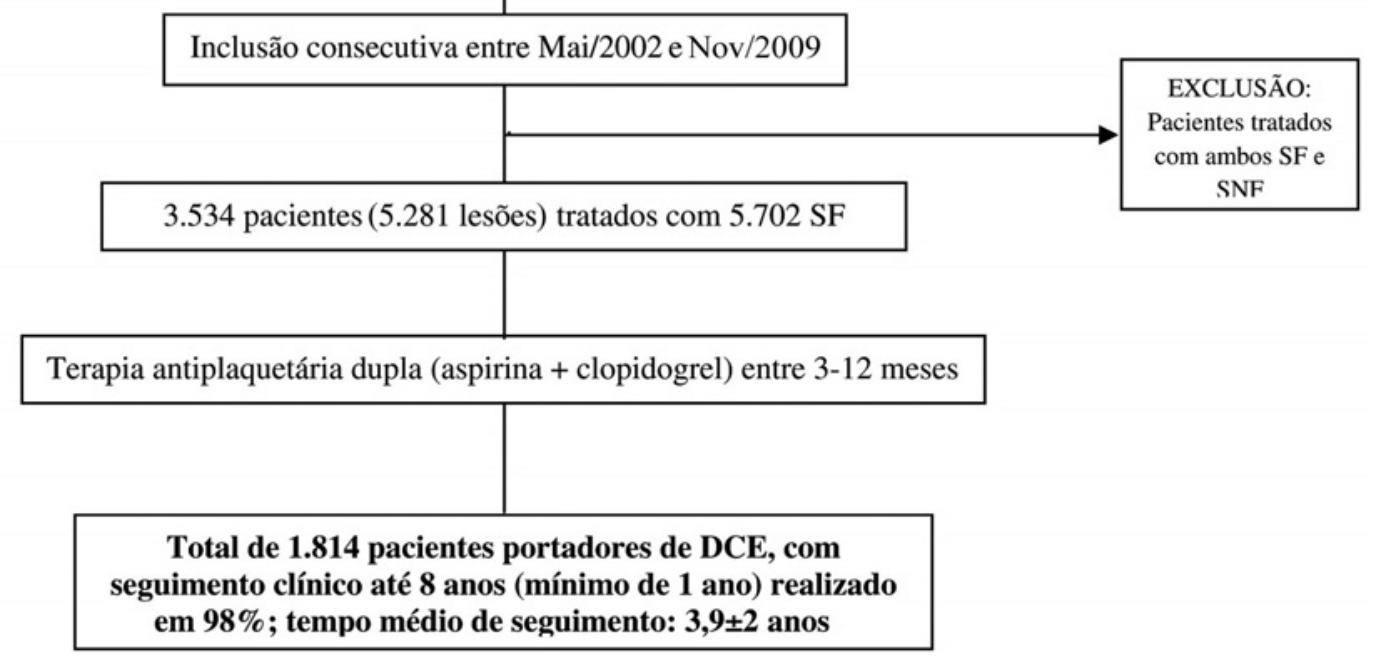

Figura 1 - Fluxograma de inclusão dos pacientes. DCE = doença coronária estável; ICP = intervenção coronária percutânea; SF = stent farmacológico; SNF = stent não-farmacológico.

indefinidamente, na dose de 100-200 mg/dia; e o clopidogrel, na dose de $75 \mathrm{mg}$ diários, era mantido inicialmente por 3 a 6 meses, até novembro de 2006 . Desde então, seu uso foi estendido para 12 meses, de acordo com as novas recomendações do Food and Drug Administration (FDA) dos Estados Unidos. ${ }^{10}$ Durante $\mathrm{O}$ procedimento, foi administrada heparina endovenosa (70$100 \mathrm{U} / \mathrm{kg}$ ) para manter o tempo de coagulação ativada $>250$ segundos (ou > 200 segundos no caso de administração de inibidor da glicoproteína Ilb/IIla).

Eletrocardiograma com 12 derivações foi obtido de rotina antes do procedimento, imediatamente após o procedimento e depois de 24 horas. Exames laboratoriais de rotina incluíram enzimas cardíacas - creatina quinase e creatina quinase fração $\mathrm{MB}(\mathrm{CK}-\mathrm{MB})$ massa pré-procedimento ( $<24$ horas), 18-24 horas pós-procedimento, e diariamente até a alta hospitalar em caso de alteração das mesmas.

\section{Análise angiográfica}

Em relação à análise angiográfica, foram obtidos estudos angiográficos seriados, incluindo angiografia pré e pós-procedimento, após a administração intracoronária de nitrato (50-200 mcg). As análises de angiografia coronária quantitativa foram realizadas por operadores experientes utilizando um sistema de análise quantitativa com detecção semiautomática das bordas luminais (QuantcorQCA-ACOM.PC versão 4.0, Siemens - Munique, Alemanha). O diâmetro luminal mínimo
(DLM) e o diâmetro de referência do vaso (DRV), obtidos pela média dos segmentos 5-10 mm proximal e distal da lesão-alvo, foram utilizados para calcular o diâmetro da estenose (DE). Assim:

$$
\mathrm{DE}=(1-\mathrm{DLM} / \mathrm{DRV}) \times 100 .
$$

Ganho imediato foi definido como a diferença do diâmetro luminal mínimo pré e pós-procedimento (DLM pós-procedimento - DLM pré-procedimento).

\section{Definições e seguimento clínico}

Os eventos cardíacos adversos maiores incluíram: morte cardíaca, infarto do miocárdio e revascularização da lesão-alvo. Como regra, todas as mortes foram consideradas cardíacas a não ser que uma causa não-cardíaca pudesse ser claramente estabelecida por estudo clínico e/ou patológico. O diagnóstico de infarto do miocárdio foi baseado no aparecimento de nova onda-Q patológica em duas ou mais derivações contíguas no eletrocardiograma e/ou elevação da CK-MB massa $>3$ vezes o limite superior da normalidade. A trombose de stent foi definida de acordo com as proposições do Academic Research Consortium (ARC): definitiva (presença de síndrome coronária aguda com confirmação angiográfica ou anatomopatológica de oclusão do stent); provável (ocorrência de morte súbita < 30 dias pósprocedimento índice ou infarto do miocárdio no território miocárdico tratado, sem confirmação angiográfica de oclusão do stent); e possível (ocorrência de morte súbita > 30 dias após o procedimento índice). A trombo- 
Tavares S, et al. Impacto de Stents Farmacológicos em Pacientes com Doença Arterial Coronária Estável Submetidos a Intervenção Coronária Percutânea na Prática Diária do Mundo Real. Rev Bras Cardiol Invasiva. 2010;18(4):392-9.

se de stent foi ainda classificada de acordo com a ocorrência temporal: aguda $(<24$ horas do procedimento), subaguda (ocorrência entre 24 horas e 30 dias pós-procedimento), tardia (ocorrência entre 1 e 12 meses pós-procedimento), e muito tardia (ocorrência $>12$ meses pós-procedimento). ${ }^{11} \mathrm{O}$ sucesso angiográfico foi definido como fluxo final TIMI 3, ausência de dissecções e estenose residual $<20 \%$ pela angiografia coronária quantitativa. O seguimento clínico foi realizado 1 mês, 6 e 12 meses após o procedimento e anualmente até o oitavo ano de acompanhamento, e consistiu de visita médica agendada ou contato telefôniCo, realizado segundo protocolo pré-definido. Todos os eventos adversos reportados, incluindo trombose de stent, foram adjudicados por um Comitê de Eventos Clínicos.

\section{Análise estatística}

As variáveis são apresentadas como frequências ou médias e desvio padrão. A probabilidade de sobrevida livre de eventos temporal foi descrita por meio de curvas de Kaplan-Meier.

\section{RESULTADOS}

Do total de 1.814 pacientes com doença coronária estável, 56,6\% eram portadores de isquemia silenciosa (Figura 2). Analisando-se as características clínicas basais, observamos que a média das idades foi de 64,2 $\pm 10,8$ anos e os pacientes tinham elevada prevalência de características de alto risco, incluindo diabetes melito em 28\%, hipertensão em 78\%, infarto do miocárdio prévio em $21 \%$, doença multiarterial em $56 \%$, e revascularização prévia em mais da metade dos pacientes $(51,7 \%)$ (Tabela 1). A Tabela 2 apresenta os dados angiográficos. A artéria descendente anterior foi o vasoalvo mais tratado (42\%), e considerando-se o total de lesões $(n=2.701) 66,3 \%$ delas eram de elevada complexidade (tipos B2/C pelos critérios do American College of Cardiology/American Heart Association).

Com relação ao procedimento, as lesões foram pré-dilatadas em 50,1\%. No total, 2.938 stents farma-

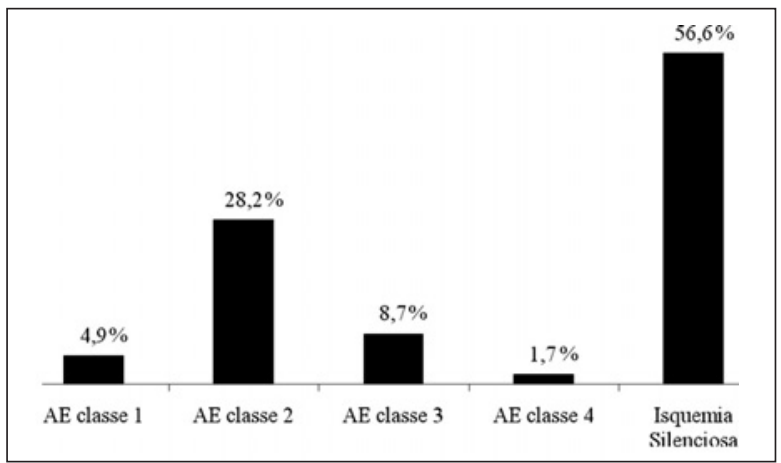

Figura 2 - Apresentação clínica de doença coronária estável (porcentual de pacientes, $\mathrm{n}=1.814$ ). $\mathrm{AE}=$ angina estável (classificada de acordo com a Canadian Cardiovascular Society).
TABELA 1

Características clínicas basais

\begin{tabular}{lc}
\hline Variável & $\mathbf{n}=\mathbf{1 . 8 1 4}$ \\
\hline Média das idades, anos & $64,2 \pm 10,8$ \\
Sexo feminino, \% & 21 \\
Hipertensão arterial sistêmica, \% & 78 \\
Diabetes melito, \% & 28 \\
Em uso de insulina & 5,4 \\
Dislipidemia, \% & 66,3 \\
Tabagismo atual, \% & 30,8 \\
História familiar de DAC, \% & 40,9 \\
IM prévio, \% & 21 \\
ICP prévia, \% & 24,8 \\
CRM prévia, \% & 26,9 \\
AVE prévio, \% & 1,5 \\
Insuficiência renal*, \% & 9,5 \\
Doença multiarterial & 56 \\
Doença vascular periférica & 3,8 \\
\hline Valores são expressos como frequência ou média e desvio \\
padrão. \\
* Definida como creatinina sérica basal $\geq 1,5$ mg/dl. \\
AVE = acidente vascular encefálico; CRM = cirurgia de revas- \\
cularização do miocárdio; DAC = doença arterial coronária; \\
ICP = intervenção coronária percutânea; IM = infarto do \\
miocárdio; $n$ = número de pacientes. \\
\hline
\end{tabular}

TABELA 2

Características angiográficas

\begin{tabular}{|c|c|}
\hline Variável & $\begin{array}{c}n=1.814 \\
(2.701 \text { lesões })\end{array}$ \\
\hline \multicolumn{2}{|l|}{ Território miocárdico acometido, \% } \\
\hline DA & 42 \\
\hline$C x$ & 29,5 \\
\hline $\mathrm{CD}$ & 27,1 \\
\hline TCE & 1,3 \\
\hline \multicolumn{2}{|l|}{ Características da lesão, \% } \\
\hline Ponte de veia safena & 5,8 \\
\hline Localização ostial & 4,6 \\
\hline Angulação > 45 graus & 5,4 \\
\hline Excentricidade & 24,1 \\
\hline Calcificação moderada ou grave & 24,5 \\
\hline Reestenose intrastent & 4,6 \\
\hline $\begin{array}{l}\text { Lesão tipo B2/C } \\
\text { (Classificação ACC/AHA) }\end{array}$ & 66,3 \\
\hline $\begin{array}{l}\text { Fração de ejeção do } \\
\text { ventrículo esquerdo < 40\%, \% }\end{array}$ & 16,2 \\
\hline \multicolumn{2}{|c|}{$\begin{array}{l}\text { Valores expressos como frequência. } \\
\text { ACC = American College of Cardiology; } \mathrm{AHA}=\text { American Heart } \\
\text { Association; } \mathrm{CD}=\text { coronária direita; } \mathrm{Cx}=\text { circunflexa; } \mathrm{DA}= \\
\text { descendente anterior; } \mathrm{n}=\text { número de pacientes; } \mathrm{TCE}=\text { tronco } \\
\text { de coronária esquerda. }\end{array}$} \\
\hline
\end{tabular}


Tavares S, et al. Impacto de Stents Farmacológicos em Pacientes com Doença Arterial Coronária Estável Submetidos a Intervenção Coronária Percutânea na Prática Diária do Mundo Real. Rev Bras Cardiol Invasiva. 2010;18(4):392-9.

cológicos foram implantados (média de 1,5 \pm 0,76 stent por paciente) e $45 \%$ dos pacientes receberam múltiplos stents. A média da pressão de liberação do stent foi de $14,7 \pm 2,8 \mathrm{~atm}$, e a pós-dilatação com cateter-balão foi aplicada em $43,7 \%$ dos casos. A Figura 3 detalha a proporção do tipo de stent utilizado, observando-se que o stent farmacológico Cypher ${ }^{\circledR}$ foi o dispositivo mais implantado $(84,4 \%)$. Ao final do procedimento, o sucesso angiográfico foi de $99 \%$. Durante a fase hospitalar, todos os pacientes foram mantidos com terapia antiplaquetária dupla e a prescrição foi mantida na alta hospitalar de acordo com o protocolo. A Tabela 3 apresenta os resultados da angiografia coronária quantitativa.

\section{Eventos clínicos}

$\mathrm{Na}$ fase intra-hospitalar, morte cardíaca ocorreu em $0,2 \%$ dos pacientes e 1,3\% evoluíram com infarto do miocárdio periprocedimento. No seguimento clínico tardio até 8 anos (média de 3,9 \pm 2 anos), a incidência cumulativa de morte cardíaca foi de 3,4\%, infarto do miocárdio em 5,5\%, e revascularização da lesão-alvo em 4,8\% dos pacientes (Figura 4). Adicionalmente, 26 pacientes apresentaram trombose de stent nesse período, e 54\% dos eventos foram classificados como trombose de stent definitiva (com confirmação angiográfica), 42\% como possível e 4\%, provável. Do

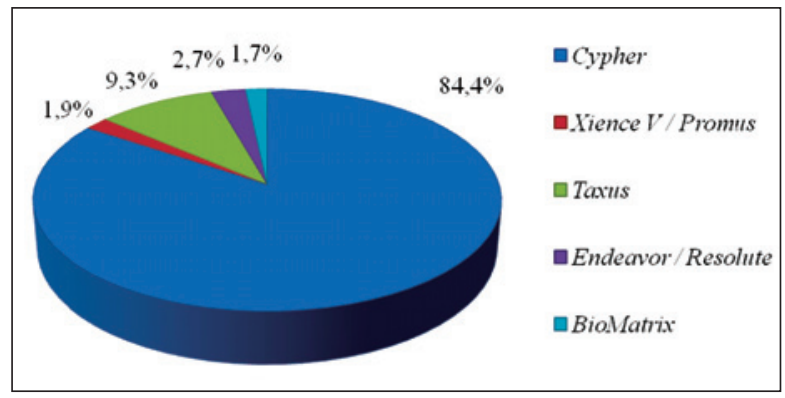

Figura 3 - Tipo de stent farmacológico implantado $(n=2.938)$.

TABELA 3

Angiografia coronária quantitativa $(n=2.701)$

\begin{tabular}{lcc}
\hline Variável & $\begin{array}{c}\text { Pré- } \\
\text { procedimento }\end{array}$ & $\begin{array}{c}\text { Pós- } \\
\text { procedimento* }\end{array}$ \\
\hline Extensão da lesão, mm & $16,4 \pm 8,3$ & - \\
DRV, mm & $2,74 \pm 0,48$ & - \\
DLM, mm & $1,24 \pm 2,52$ & $2,95 \pm 2,34$ \\
DE, \% & $65,3 \pm 13,8$ & $5 \pm 3,7$ \\
Ganho imediato, $\mathrm{mm}$ & - & $1,9 \pm 0,46$ \\
\hline
\end{tabular}

Valores expressos como média e desvio padrão.

* Análise do segmento intrastent.

$\mathrm{DE}=$ diâmetro da estenose; $\mathrm{DLM}=$ diâmetro luminal

mínimo; DRV = diâmetro de referência do vaso. total de tromboses de stent definitiva e provável (0,8\%), dois terços ocorreram após um ano. A Figura 5 demonstra as curvas de sobrevida livre de eventos (KaplanMeier) no seguimento tardio até 8 anos.

\section{DISCUSSÃO}

Na presente análise, podemos observar que pacientes de mundo real portadores de doença coronária estável tratados inicialmente com stent farmacológico apresentaram excelente evolução clínica no seguimento a longo prazo, incluindo baixa mortalidade cardíaca e segurança mantida (trombose de stent definitiva/provável < 1\%). Os stents farmacológicos ainda demonstraram efetividade clínica sustentada, incluindo incidência de revascularização da lesão-alvo $<5 \%$ no seguimento médio de 3,9 anos, e sobrevida livre de revascularização da lesão-alvo > 90\% no acompanhamento até 8 anos.

De maneira geral, os stents farmacológicos demonstraram superioridade em relação aos stents nãofarmacológicos, principalmente pela marcante redução das taxas de revascularização da lesão-alvo. ${ }^{3-5} \mathrm{Um}$ estudo de Stone et al. ${ }^{6}$ reportou a evolução clínica tardia (4 anos) de 5.261 pacientes (doença coronária estável em 62\%) incluídos em ensaios clínicos randomizados que compararam stents farmacológicos de primeira geração $\left(\right.$ Cypher $^{\circledR}$ e Taxus $^{\circledR}$ ) vs. stents nãofarmacológicos. Nessa análise, não se evidenciaram diferenças significativas entre stents farmacológicos e stents não-farmacológicos nos desfechos clínicos de morte por todas as causas $\left(\right.$ Cypher $^{\circledR} 6,7 \%$ vs. stents não-farmacológicos 5,3\%, P = NS; Taxus ${ }^{\circledR} 6,1 \%$ vs. stents não-farmacológicos $6,6 \%$, P = NS) e infarto do miocárdio (Cypher ${ }^{\circledR} 6,4 \%$ vs. stents não-farmacológicos $6,2 \%, P=N S ;$ Taxus $^{\circledR} 7 \%$ vs. stents não-farmacológicos $6,3 \%, P=N S]$. No entanto, os stents farmacológicos estiveram associados a menores taxas de revascularização da lesão-alvo (Cypher ${ }^{\circledR} 7,8 \%$ vs. stents não-farmacológicos 23,6\%, P < 0,001; Taxus ${ }^{\circledR} 10,1 \%$ vs. stents não-farmacológicos 20\%, P $<0,001) .{ }^{6}$ Tais achados têm sido confirmados na prática diária do mundo real. Em um estudo reportado por Wenaweser et al. ${ }^{12}$, incluindo 8.146 pacientes complexos tratados com stents farmacológicos em dois centros clínicos, as taxas de mortalidade total e de infarto do miocárdio aos 4 anos de seguimento clínico foram, respectivamente, de 10,6\% e $4,6 \%$. Similarmente, resultados da população geral

\begin{tabular}{|ccccc|}
\hline $0,2 \% \frac{3,4 \%}{3,5 \%}$ & & $4,8 \%$ & \\
\hline Morte Cardiaca & IM & RLA & TS (Def./Prov.) \\
\hline
\end{tabular}

Figura 4 - Taxas cumulativas de eventos adversos na fase intrahospitalar (cinza) e no seguimento tardio (preto). IM = infarto do miocárdio; RLA = revascularização da lesão-alvo; TS (Def./Prov.) = trombose de stent definitiva/provável. 
Tavares S, et al. Impacto de Stents Farmacológicos em Pacientes com Doença Arterial Coronária Estável Submetidos a Intervenção Coronária Percutânea na Prática Diária do Mundo Real. Rev Bras Cardiol Invasiva. 2010;18(4):392-9.

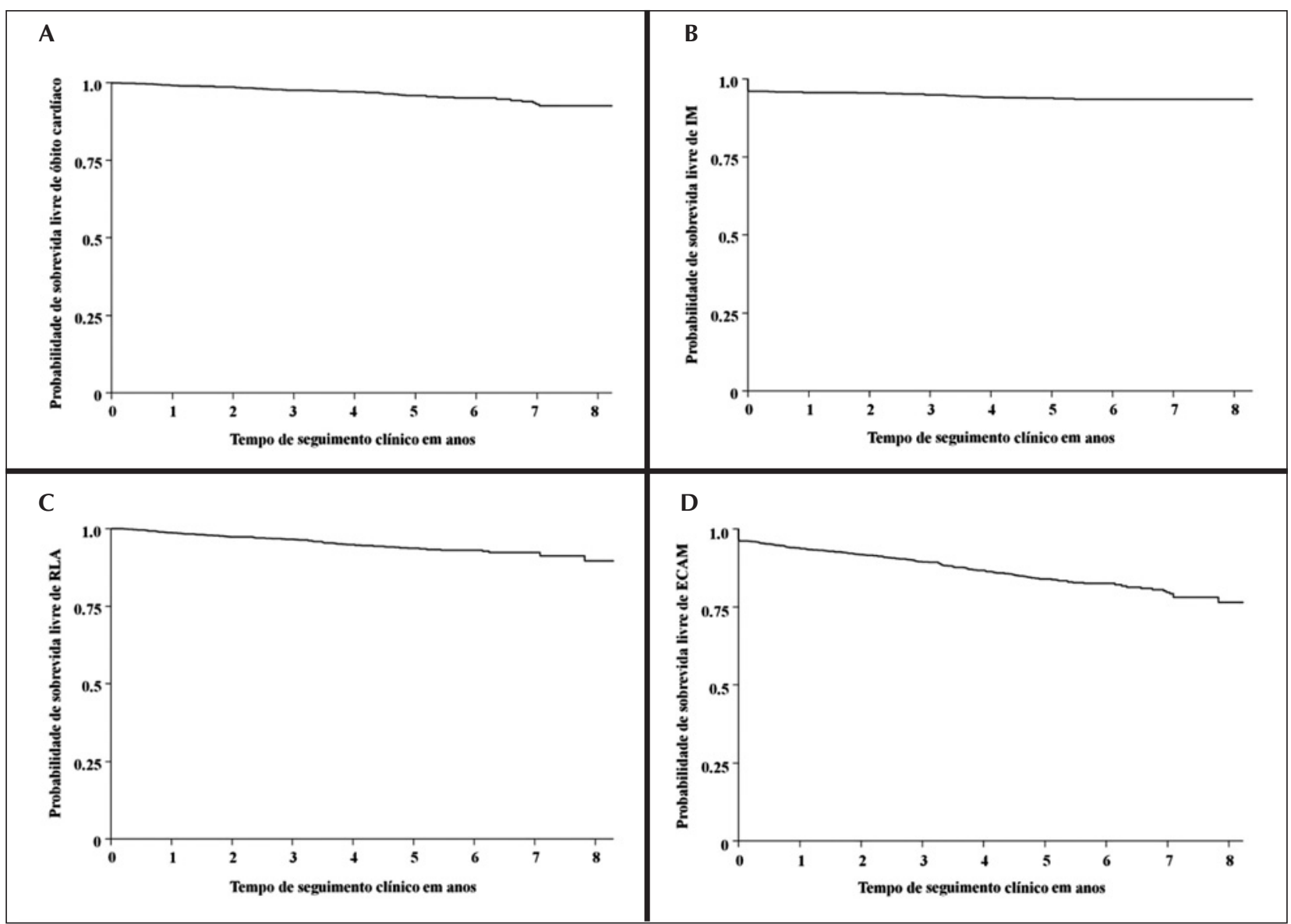

Figura 5 - Curvas de Kaplan-Meier de sobrevida livre de eventos adversos até 8 anos de seguimento clínico Em A, sobrevida livre de óbito cardíaco. Em B, sobrevida livre de IM. Em C, sobrevida livre de RLA. Em D, sobrevida livre de ECAM. ECAM = eventos cardíacos adversos maiores; $I M=$ infarto do miocárdio; RLA = revascularização da lesão-alvo.

do Registro DESIRE (doença coronária estável em 59\%, síndrome coronária aguda em $41 \%$ ) com seguimento $>4$ anos (mediana de 5 anos) demonstraram baixas taxas de morte cardíaca, infarto do miocárdio e revascularização da lesão-alvo $(5,6 \%, 3,2 \%$, e $6,6 \%$, respectivamente) no acompanhamento tardio. ${ }^{13}$

No entanto, o papel da intervenção coronária percutânea em pacientes com doença coronária estável permanece controverso. Schomig et al. ${ }^{14}$ reportaram uma meta-análise envolvendo 7.513 pacientes incluídos em 17 ensaios clínicos randomizados, e concluíram que a intervenção coronária percutânea em pacientes com angina estável tinha impacto em termos de mortalidade vs. tratamento clínico isolado. Nesse estudo, a intervenção coronária percutânea esteve associada a redução significativa de mortalidade total [odds ratio (OR) 0,80; intervalo de confiança de 95\% (IC 95\%) 0,63-0,99] e diminuições não-significativas de morte cardíaca (OR 0,74; IC 95\% 0,51-1,06) e de infarto do miocárdio (OR 0,90; IC 95\% 0,66-1,23). ${ }^{14}$ Já no ensaio clínico randomizado COURAGE, que comparou 1.149 pacientes submetidos inicialmente a intervenção coro- nária percutânea associada a terapia medicamentosa otimizada vs. 1.138 pacientes tratados com terapia medicamentosa otimizada, não houve diferença no desfecho primário de morte por todas as causas/infarto do miocárdio não-fatal no seguimento de 4,6 anos (19\% vs. $18,5 \%$, respectivamente; $\mathrm{P}=\mathrm{NS})$. No entanto, um terço dos pacientes alocados no grupo tratado com terapia medicamentosa otimizada foi submetido a procedimento de revascularização coronária vs. 21,2\% de revascularização da lesão-alvo no grupo tratado com intervenção coronária percutânea associada a terapia medicamentosa otimizada $(P<0,001)$. Em nossa subanálise do Registro DESIRE, a incidência de morte cardíaca/infarto agudo do miocárdio não-fatal no seguimento médio de 3,9 anos foi de 8,9\% (morte cardíaca, 3,4\%; infarto agudo do miocárdio, 5,5\%) e a revascularização da lesão-alvo foi de 4,8\%. Comparados aos subgrupos do estudo COURAGE, tais achados sugerem grande benefício do stent farmacológico nessa população de doença coronária estável, já que no estudo COURAGE: apenas $2,7 \%$ dos pacientes submetidos a intervenção coronária percutânea receberam stent farmacológico; a taxa de inclusão $(<1$ pa- 
ciente/centro/mês durante aproximadamente 5 anos) sugere superseleção dos pacientes, potencialmente com lesões menos complexas; e $32,6 \%$ dos pacientes tratados inicialmente com estratégia conservadora foram submetidos a intervenção coronária percutânea (maioria no primeiro ano). Além disso, a taxa de sucesso angiográfico/procedimento percutâneo relativamente baixa no estudo COURAGE ( 90\%) pode ter impactado de maneira negativa os resultados do braço percutâneo. ${ }^{1,15}$ No subgrupo com doença coronária estável no Registro DESIRE, que incluiu pacientes do mundo real de moderada complexidade, a taxa de sucesso angiográfico foi de $99 \%$, a despeito de elevada prevalência de lesões complexas (66,3\%, tipo B2/C) (Tabela 2). A Tabela 4 apresenta a comparação das características basais, do procedimento e dos desfechos clínicos do Registro DESIRE vs. estudo COURAGE. Quando colocados em perspectiva, nossos resultados sugerem que os stents farmacológicos podem oferecer benefícios significativos se utilizados como estratégia de tratamento inicial em pacientes portadores de doença coronária estável, principalmente pela diminuição expressiva da revascularização da lesão-alvo em fun- ção da eficácia dos stents farmacológicos na inibição da formação de hiperplasia neointimal e reestenose. Isso, obviamente, não exclui a necessidade de otimização do tratamento farmacológico adjunto, o que pode promover benefícios adicionais (além do stent farmacológico) nessa população. No entanto, novos estudos randomizados comparando diferentes estratégias de tratamento na doença coronária estável, incluindo stent farmacológico, são necessários para confirmar essa hipótese.

\section{Limitações do estudo}

Este estudo representa a experiência clínica de uma instituição em que stents farmacológicos têm sido utilizados como estratégia preferencial em pacientes com indicação de intervenção coronária percutânea. Como o Registro DESIRE conta com inclusão contínua de pacientes por tempo indeterminado, a análise atual reflete os achados referentes a um período preestabelecido (maio de 2002 a novembro de 2009). No período do estudo, foram excluídos os pacientes tratados com stents não-farmacológicos ou com a combinação de stents farmacológicos e stents não-farmacológicos. Por último, os diferentes tipos de stent far-

TABELA 4

Comparação das características basais/procedimento e dos resultados clínicos de pacientes com doença coronária estável incluídos no Registro DESIRE vs. estudo COURAGE

\begin{tabular}{|c|c|c|c|}
\hline \multirow[b]{2}{*}{ Tratamento } & \multirow{2}{*}{$\begin{array}{c}\text { DESIRE DCE } \\
\text { SF }\end{array}$} & \multicolumn{2}{|c|}{ COURAGE } \\
\hline & & $\mathrm{TMO}+\mathrm{SNF}$ & TMO \\
\hline$n$ & 1.814 & $1.149 *$ & 1.138 \\
\hline Média das idades, anos & $64,2 \pm 10,8$ & $61,5 \pm 10,1$ & $61,8 \pm 9,7$ \\
\hline Diabete melito, \% & 28,7 & 31,9 & 35,1 \\
\hline \multicolumn{4}{|l|}{ Apresentação clínica, \% } \\
\hline AE classe $0 / 1$ & 61,5 & 41,3 & 43 \\
\hline AE classe $2 / 3$ & 36,9 & 58,3 & 56,8 \\
\hline Doença multiarterial, \% & 56,4 & 68,5 & 69,8 \\
\hline Número de lesões tratadas & 2.701 & 1.688 & - \\
\hline Vaso-alvo: DA proximal, \% & 35 & 31,3 & 36,6 \\
\hline Implante de múltiplos stents, \% & 44,8 & 36,2 & - \\
\hline Sucesso angiográfico, \% & 99 & 93,4 & - \\
\hline Sucesso clínico/procedimento, \% & 98,4 & 89 & - \\
\hline Tempo de seguimento, anos & $3,9^{+}$ & $4,6^{\ddagger}$ & $4,6^{\ddagger}$ \\
\hline Pacientes com seguimento, \% & 98 & 90,7 & 91,5 \\
\hline \multicolumn{4}{|l|}{ Desfechos clínicos tardios, \% } \\
\hline Morte por qualquer causa & 7,4 & 7,6 & 8,3 \\
\hline IM & 5,5 & 13,2 & 12,3 \\
\hline Nova revascularização & $4,8^{\S} / 8,5^{\prime \prime}$ & 21,1 & 32,6 \\
\hline TS (definitiva/provável) & 0,8 & - & - \\
\hline
\end{tabular}

Valores são expressos como frequência ou média e desvio padrão.

* 6,4\% não foram submetidos a intervenção. ${ }^{+}$Média. ${ }^{*}$ Mediana. ${ }^{\S}$ RLA. " Revascularização de vaso-alvo (não-RLA) ou de vaso não-alvo. $\mathrm{AE}=$ angina estável; $\mathrm{DA}=$ descendente anterior; $\mathrm{DCE}=$ doença coronária estável; $\mathrm{IM}=$ infarto do miocárdio; $\mathrm{n}=$ número de pacientes; RLA = revascularização da lesão-alvo; SF = stent farmacológico; SNF = stent não-farmacológico; TS = trombose de stent. 
Tavares S, et al. Impacto de Stents Farmacológicos em Pacientes com Doença Arterial Coronária Estável Submetidos a Intervenção Coronária Percutânea na Prática Diária do Mundo Real. Rev Bras Cardiol Invasiva. 2010;18(4):392-9.

macológico foram implantados em proporções nãobalanceadas, e as indicações foram baseadas em disponibilidade, anatomia/localização da lesão, quadro clínico e/ou preferência do operador.

\section{CONCLUSÃO}

Nessa subanálise do Registro DESIRE envolvendo pacientes do mundo real portadores de doença coronária estável, a utilização de stents farmacológicos esteve associada a excelente evolução clínica tardia, incluindo taxa de revascularização da lesão-alvo $<5 \%$ e segurança sustentada. Comparados a dados históricos, tais achados demonstram grande benefício do stent farmacológico nessa população, sugerindo que a intervenção coronária percutânea com stent farmacológico pode ser uma alternativa inicial segura e eficaz no tratamento de pacientes com doença coronária estável.

\section{CONFLITO DE INTERESSES}

Os autores declararam inexistência de conflito de interesses relacionado a este manuscrito.

\section{REFERÊNCIAS}

1. Boden WE, O'Rourke RA, Teo KK, Hartigan PM, Maron DJ, Kostuk WJ, et al. Optimal medical therapy with or without PCI for stable coronary disease. N Engl J Med. 2007;356(15): 1503-16.

2. O'Rourke RA. Optimal medical therapy is a proven option for chronic stable angina. J Am Coll Cardiol. 2008;52(11):905-7.

3. Moses JW, Leon MB, Popma JJ, Fitzgerald PJ, Holmes DR, O'Shaughnessy C, et al. Sirolimus-eluting stents versus standard stents in patients with stenosis in a native coronary artery. N Engl J Med. 2003;349(14):1315-23.

4. Stone GW, Ellis SG, Cox DA, Hermiller J, O'Shaughnessy C, Mann JT, et al. A polymer-based, paclitaxel-eluting stent in patients with coronary artery disease. N Engl J Med. 2004; 350(3):221-31.

5. Kirtane AJ, Gupta A, lyengar S, Moses JW, Leon MB, Applegate $R$, et al. Safety and efficacy of drug-eluting and bare metal stents: comprehensive meta-analysis of randomized trials and observational studies. Circulation. 2009;119(25):3198-206.
6. Stone GW, Moses JW, Ellis SG, Schofer J, Dawkins KD, Morice MC, et al. Safety and efficacy of sirolimus- and paclitaxel-eluting coronary stents. N Engl J Med. 2007;356(10): 998-1008.

7. Sousa A, Costa JR Jr, Moreira AC, Cano M, Maldonado G, Costa RA, et al. Long-term clinical outcomes of the DrugEluting Stents in the Real World (DESIRE) Registry. J Interv Cardiol. 2008;21(4):307-14.

8. Kaul P, Naylor CD, Armstrong PW, Mark DB, Theroux P, Dagenais GR. Assessment of activity status and survival according to the Canadian Cardiovascular Society angina classification. Can J Cardiol. 2009;25(7):e225-31.

9. Mattos LA, Lemos PA. Diretrizes da Sociedade Brasileira de Cardiologia - Intervenção Coronária Percutânea e Métodos Adjuntos Diagnósticos em Cardiologia Intervencionista (II Edição - 2008). Arq Bras Cardiol. 2008;91(6 Supl 1):1-58.

10. Grines $\mathrm{CL}$, Bonow RO, Casey DE Jr, Gardner TJ, Lockhart PB, Moliterno DJ, et al. Prevention of premature discontinuation of dual antiplatelet therapy in patients with coronary artery stents: a science advisory from the American Heart Association, American College of Cardiology, Society for Cardiovascular Angiography and Interventions, American College of Surgeons, and American Dental Association, with representation from the American College of Physicians. Circulation. 2007;115(6): 813-8.

11. Cutlip DE, Windecker S, Mehran R, Boam A, Cohen DJ, van Es GA, et al. Clinical end points in coronary stent trials: a case for standardized definitions. Circulation. 2007;115(17): 2344-51.

12. Wenaweser $P$, Daemen J, Zwahlen $M$, van Domburg R, Juni $P$, Vaina $S$, et al. Incidence and correlates of drug-eluting stent thrombosis in routine clinical practice. 4-year results from a large 2-institutional cohort study. J Am Coll Cardiol. 2008;52(14):1134-40.

13. Costa JR Jr, Sousa A, Moreira AC, Costa RA, Cano M, Maldonado $G$, et al. Incidence and predictors of very late ( $>$ or $=4$ years) major cardiac adverse events in the DESIRE (Drug-Eluting Stents in the Real World)-Late registry. JACC Cardiovasc Interv. 2010;3(1):12-8.

14. Schomig A, Mehilli J, de Waha A, Seyfarth M, Pache J, Kastrati A. A meta-analysis of 17 randomized trials of a percutaneous coronary intervention-based strategy in patients with stable coronary artery disease. J Am Coll Cardiol. 2008; 52(11):894-904.

15. Kereiakes DJ, Teirstein PS, Sarembock IJ, Holmes DR Jr, Krucoff MW, O'Neill WW, et al. The truth and consequences of the COURAGE trial. J Am Coll Cardiol. 2007;50(16):1598-603. 